\title{
PROBABILISTIC METHODS FOR A LINEAR REACTION-HYPERBOLIC SYSTEM WITH CONSTANT COEFFICIENTS
}

\author{
By ElizaBeth A. BRooks \\ National Institute of Environmental Health Sciences

\begin{abstract}
Linear reaction-hyperbolic systems of partial differential equations in one space dimension arise in the study of the physiological process by which materials are transported in nerve cell axons. Probabilistic methods are developed to derive a closed form approximate solution for an initial-boundary value problem of such a system. The approximate solution obtained is a translating solution of a heat equation. An estimate is proved giving the deviation of this approximate traveling wave solution from the exact solution.
\end{abstract}

1. Introduction. In this paper we show how a probabilistic model can be used to derive an approximate closed-form solution to an initial-boundary value problem for a simple linear, constant-coefficient hyperbolic system of equations in one space dimension. Subsequent papers will detail how these probabilistic techniques have been extended to more general linear hyperbolic systems with both constant and nonconstant coefficients.

While other papers have concentrated on connecting Markovian random processes with corresponding governing hyperbolic equations [Goldstein (1951), Kac (1974), Bartlett (1957, 1978), Cane (1967, 1975), Kaplan (1964), Orsingher (1985, 1990), Orsingher and Bassan (1992), Kolesnik (1998)], the significance of the work presented here is the actual obtaining of the approximate solution for the hyperbolic system by using its related random process.

The equations investigated are motivated by a biological problem; hence, we first describe how this class of equations arises in modeling transport processes in nerve cells. In nerve cells, protein synthesis occurs only in the cell body (soma); thus, the proteins, membrane-bound neurotransmitters and other essential structural elements must be transported down the axon. Since the mid-1980's, Reed and Blum have been formulating mathematical models of fast axonal transport [Blum and Reed (1985), Reed and Blum (1986)]. Each of their models is of the form $L u=f(u)$ where $u(x, t)$ is a vector of chemical concentrations, $x$ represents distance along the axon, $t$ represents time, $L$ is a linear hyperbolic operator and $f$ is a linear or nonlinear mapping representing the interactions of the various chemical constituents.

An important special class of equations arises when a single species is transported into a spatially homogeneous axon. The resulting linear, constantcoefficient equations do not have traveling wave solutions which approach

Received May 1998; revised November 1998.

AMS 1991 subject classifications. Primary 0G99, 35L45, 35L50; secondary 92C20.

Key words and phrases. Hyperbolic equations, stochastic processes, traveling waves, renewal theory, central limit theorem. 
constant states as $x \rightarrow \infty$ and $x \rightarrow-\infty$; however, Reed, Venakides and Blum (1990) showed through a nonrigorous singular perturbation analysis that as the rates of the chemical reactions approach $\infty$, the solutions of this general class of hyperbolic equations approach traveling wave solutions of a parabolic heat equation. The probabilistic methods developed here allow us to show rigorously this interesting result for the simplest linear model addressed in Reed, Venakides and Blum (1990) and to give the deviation of the approximate traveling wave solution from the exact solution. Furthermore, the probabilistic techniques provide an intuitive and elegant explanation of why solutions of a general class of hyperbolic equations look like "spreading" normal density curves.

In Section 2 we present the intuition for the probabilistic methods developed for the hyperbolic system describing a simple transport model. A homogeneous stochastic process is defined which evolves according to the partial differential equations. The approximate solution obtained is an approximate mass density of this stochastic process. We derive the approximate mass density using techniques similar to (but distinct from) renewal theory as discussed in Feller (1968), Karlin and Taylor (1975) and Cox (1967).

In Section 3, we present the local central limit theorem and large deviation estimates necessary for the proofs of the results discussed intuitively in the previous section. Finally, in Section 4 we prove that the approximate solution of our simple transport model is actually a translating solution of a heat equation and we determine its deviation from the exact solution.

The idea of the probabilistic approach for hyperbolic equations of this kind is due to Gregory F. Lawler [Example 5 on pages 110 and 111 of Lawler (1995)].

2. The idea of the probabilistic methods. Consider the following initial-boundary value problem:

$$
\begin{aligned}
\frac{\partial p}{\partial t} & =-k_{1} p+k_{2} q, \\
\frac{\partial q}{\partial t}+v \frac{\partial q}{\partial x} & =k_{1} p-k_{2} q
\end{aligned}
$$

with initial conditions

$$
p(x, 0)=q(x, 0)=0, \quad x>0
$$

and boundary condition

$$
q(0, t)=q_{0}, \quad t \geq 0
$$

where $q_{0}, v, k_{1}$, and $k_{2}$ are positive constants.

This system of equations describes a simple transport model in which particles of type $p$ change to particles of type $q$ at rate $k_{1}$, and particles of type $q$ change to type $p$ at rate $k_{2}$. Particles of type $p$ are stationary while those of type $q$ move with velocity $v$. We can think of $p(x, t)$ and $q(x, t)$ as representing the concentration of particles of type $p$ and $q$, respectively, at position $x$ at time $t$. From the initial and boundary conditions, we see that the initial 
concentration of $p$ and $q$ particles is 0 for all $x>0$ and there is a continual influx of $q$ particles at $x=0$.

To formulate this system of PDE's probabilistically, first note that since the system is linear, the particles evolve independently. We begin by considering the evolution of a single particle as described by (1)-(4).

The evolution of a single particle can be described by a stochastic process $Z_{t}=\left(X_{t}, C_{t}\right)$, where $X_{t}$ is the position of the particle at time $t$, and $C_{t}$ is the state of the particle ( $p$ or $q$ ) at time $t$. As dictated by the boundary condition (4), $Z_{0}=(0, q)$. Furthermore, note that $X_{t}=v Y_{t}$ where $Y_{t}$ is the amount of time spent in state $q$ up through time $t$.

We give here an intuitive argument [Lawler (1995)] to show that $Y_{t}$ is approximately normal with mean $\mu t$ and variance $\sigma^{2} t$, where $\mu=k_{1}\left(k_{1}+k_{2}\right)^{-1}$ and $\sigma^{2}=2 k_{1} k_{2}\left(k_{1}+k_{2}\right)^{-3}$.

If we define random variables

$$
\begin{aligned}
& Q_{1} \equiv \inf \left\{t>0: C_{t} \neq q\right\}, \\
& P_{1} \equiv \inf \left\{t>0: C_{Q(1)+t}=q\right\}, \\
& T_{1}=Q_{1}+P_{1},
\end{aligned}
$$

and for $i>1$,

$$
\begin{aligned}
Q_{i} & \equiv \inf \left\{t>0: C_{T(1)+\cdots+T(i-1)+t} \neq q\right\}, \\
P_{i} & \equiv \inf \left\{t>0: C_{T(1)+\cdots+T(i-1)+Q(i)+t}=q\right\}, \\
T_{i} & =Q_{i}+P_{i},
\end{aligned}
$$

then $Q_{i}$ and $P_{i}$ give the amount of time spent in states $q$ and $p$, respectively, upon the $i$ th visit to those states. Furthermore, $Q_{1}, \ldots, Q_{n}$ are independent, exponential random variables with rate parameter $k_{2}$ and hence have mean $\mu_{q}=k_{2}^{-1}$ and standard deviation $\sigma_{q}=k_{2}^{-1}$. Similarly, $P_{1}, \ldots, P_{n}$ are independent, exponential random variables with mean $\mu_{p}=k_{1}^{-1}$ and standard deviation $\sigma_{p}=k_{1}^{-1}$. Notice that

$$
Y_{t}=Q_{1}+\cdots+Q_{N(t)}+\min \left(Q_{N(t)+1}, t-\left(T_{1}+\cdots+T_{N(t)}\right)\right),
$$

where $N(t)=\sup \left\{n>0: T_{1}+\cdots+T_{n} \leq t\right\}$ counts the number of transitions of the particle from state $p$ to state $q$ in time $t$.

By the central limit theorem, we know that $Q_{1}+\cdots+Q_{n}$ is approximately normal with mean and variance $n \mu_{q}$; similarly, for $P_{1}+\cdots+P_{n}$. Furthermore, we expect $N(t)$ to be approximately $t\left(\mu_{p}+\mu_{q}\right)^{-1}$. Finally, for large $s$, the amount of time spent in state $q$ in $[s, s+\Delta s]$ is approximately $\mu_{q}\left(\mu_{q}+\mu_{p}\right)^{-1} \Delta s$; therefore, we can approximate $\min \left(Q_{n+1}, t-\left(T_{1}+\cdots+T_{n}\right)\right)$, the amount of time spent in state $q$ between times $T_{1}+\cdots+T_{n}$ and $t$, by $\mu_{q}\left(\mu_{p}+\mu_{q}\right)^{-1}(t-$ $\left.\left(T_{1}+\cdots+T_{n}\right)\right)$.

Using the above approximations in (8) it is straightforward that

$$
Y_{t} \approx \mu t+\sigma \sqrt{t} B
$$


where $\mu=k_{1}\left(k_{1}+k_{2}\right)^{-1}, \sigma^{2}=2 k_{1} k_{2}\left(k_{1}+k_{2}\right)^{-3}$, and $B$ is a standard normal random variable. The following result, which will be proved in Section 4 , gives the deviation of the density of this normal approximation from the actual density of $Y_{t}$.

THEOREM 2.1. For $x \in[\mu t / 2,(\mu+1) t / 2], f_{Y(t)}(x)=(d / d x) P\left\{Y_{t} \leq x\right\}$ is given by

$$
\begin{aligned}
f_{Y(t)}(x)=( & \left.+O\left(t^{-1 / 2}\right)\right) \frac{1}{\sigma \sqrt{t}} \eta\left(\frac{x-\mu t}{\sigma \sqrt{t}}\right) \\
+ & \frac{1}{\sqrt{\left.k_{1}(t-x)+k_{2} x\right)}} O\left(t^{-1 / 2}\right)\left|\frac{k_{1}(t-x)-k_{2} x}{\sqrt{k_{2} x+k_{1}(t-x)}}\right|^{4} \\
& \times \eta\left(\frac{k_{1}(t-x)-k_{2} x}{\sqrt{k_{2} x+k_{1}(t-x)}}\right) \\
+ & o\left(t^{-3 / 2}\right),
\end{aligned}
$$

where $\eta$ is the density of a standard normal random variable,

$$
\mu=\frac{k_{1}}{k_{1}+k_{2}}
$$

and

$$
\sigma^{2}=\frac{2 k_{1} k_{2}}{\left(k_{1}+k_{2}\right)^{3}}
$$

Since $X_{t}=v Y_{t}$, the density of $X_{t}$ follows immediately from Theorem 2.1.

Corollary 2.2. For $x \in[\mu v t / 2,(\mu+1) v t / 2], f_{X(t)}(x)=(d / d x) P\left\{X_{t} \leq x\right\}$ is given by

$$
\begin{aligned}
f_{X(t)}(x)= & v^{-1}\left(1+O\left(t^{-1 / 2}\right)\right) \frac{1}{\sigma \sqrt{t}} \eta\left(\frac{x v^{-1}-\mu t}{\sigma \sqrt{t}}\right) \\
& +v^{-1} \frac{1}{\sqrt{\left.k_{1}\left(t-x v^{-1}\right)+k_{2} x v^{-1}\right)}} O\left(t^{-1 / 2}\right)\left|\frac{k_{1}\left(t-x v^{-1}\right)-k_{2} x v^{-1}}{\sqrt{k_{2} x v^{-1}+k_{1}\left(t-x v^{-1}\right)}}\right|^{4} \\
& \times \eta\left(\frac{k_{1}\left(t-x v^{-1}\right)-k_{2} x v^{-1}}{\sqrt{k_{2} x v^{-1}+k_{1}\left(t-x v^{-1}\right)}}\right) \\
& +v^{-1} o\left(t^{-3 / 2}\right),
\end{aligned}
$$

where $\eta, \mu$ and $\sigma^{2}$ are as defined in Theorem 2.1.

Finally, to make the correspondence with initial-boundary value problem (1)-(4), let us suppose that $q_{0} v$ particles per unit time enter at $x=0$ in state $q$ from time $t=0$ until the present time $T$. Since the equations are linear, 
the particles behave independently; thus, to find the mass density $p(x, T)+$ $q(x, T)$, we simply add the individual densities to conclude that

$$
p(x, T)+q(x, T)=q_{0} v \int_{0}^{T} f_{X(t)}(x) d t .
$$

In Section 4 we will use Corollary 2.2 and probabilistic large deviation estimates to obtain the following theorem from (13).

THEOREM 2.3.

$$
p(x, T)+q(x, T)=q_{0} \mu^{-1} H\left(v^{-1}(x-\mu v T), T\right)+O\left(T^{-1 / 2}\right),
$$

where

$$
H(s, t)=\int_{-\infty}^{0} \frac{1}{\sqrt{2 \pi \sigma^{2} t}} \exp \left(\frac{-(s-y)^{2}}{2 \sigma^{2} t}\right) d y
$$

is the solution of the following initial value problem for the heat equation

$$
\begin{aligned}
\frac{\partial H}{\partial t} & =\frac{1}{2} \sigma^{2} \frac{\partial^{2} H}{\partial s^{2}} \\
H(s, 0) & = \begin{cases}1, & \text { for } s \leq 0 \\
0, & \text { for } s>0\end{cases}
\end{aligned}
$$

and where $\mu$ and $\sigma^{2}$ are given by (10) and (11), respectively. tion

Parenthetically, $u(x, T)=p(x, T)+q(x, T)$ is also the solution of the equa-

$$
\frac{\partial^{2} u}{\partial T^{2}}+v \frac{\partial^{2} u}{\partial x \partial T}+k_{1} v \frac{\partial u}{\partial x}+\left(k_{1}+k_{2}\right) \frac{\partial u}{\partial T}=0,
$$

which is the governing second-order PDE of a one-dimensional model of random evolution discussed in Kolesnik (1998).

3. Central limit theorem and large deviation estimates. In this section we present local central limit theorem and large deviation estimates that will be needed in the proofs of the following section. We begin with some necessary definitions.

Suppose $X_{1}, X_{2}, \ldots$ are independent random variables with the same lattice distribution with step 1 ; that is, $P\left\{X_{i} \in \mathbb{Z}\right\}=1$, but $P\left\{X_{i} \in j \mathbb{Z}\right\}<1$ for all $j>1$. We shall denote by $F$ the probability distribution of $X_{i}$. The $k$ th moment of $X_{i}$ will be denoted by $\bar{\mu}_{k}=E\left(X_{i}^{k}\right)$. We will suppose $\bar{\mu}_{1}=0$ and put $\bar{\mu}_{2}=\bar{\sigma}^{2}$. Finally, we will denote the density function of a standard normal random variable by $\eta$. The density function of a random variable $X$ will be denoted by $f_{X}$.

We will use the following result given in Section 4.5 of Ibragimov and Linnik (1971). 
THEOREM 3.1. Let $X_{1}, X_{2}, \ldots$ be independent, identically distributed lattice random variables with step 1 . If $\bar{\mu}_{k}<\infty$ for some $k \geq 3$,

$$
P\left\{\frac{X_{1}+\cdots+X_{n}}{\bar{\sigma} \sqrt{n}}=j\right\}=\frac{1}{\bar{\sigma} \sqrt{n}}\left[\eta(j)+\sum_{i=1}^{k-2} n^{(-1 / 2) i} P_{i}(j) \eta(j)+o\left(n^{(-1 / 2)(k-1)}\right)\right] .
$$

Here $P_{i}$ is a real polynomial of degree $i$ depending only on $\bar{\mu}_{1}, \ldots, \bar{\mu}_{k}$ but not on $n$ and $k$ (or otherwise on $F$ ).

We will only need to use the following corollary.

Corollary 3.2. Under the conditions of Theorem 3.1 , if $\bar{\mu}_{5}<\infty$,

$$
P\left\{\frac{X_{1}+\cdots+X_{n}}{\bar{\sigma} \sqrt{n}}=j\right\}=\frac{1}{\bar{\sigma} \sqrt{n}}\left[\eta(j)+O\left(n^{-1 / 2}\right)|j|^{4} \eta(j)+o\left(n^{-2}\right)\right] .
$$

Recall that if $Y$ is a Poisson random variable with parameter $m$, then

$$
P\{Y=n\}=e^{-m} \frac{m^{n}}{n !} .
$$

We can easily see that $Y$ has mean $m$, variance $m$, and that the fifth moment of $Y$ is finite. Recognizing that a Poisson random variable with integer parameter $m$ is the sum of $m$ Poisson random variables with parameter 1 , the following lemma can be obtained from Corollary 3.2. (For noninteger $m$, we can rewrite $Y$ as $Y=Y_{1}+Y_{2}$, where $Y_{1}$ has parameter $[m]$ and $Y_{2}$ has parameter $m-[m]$.

LEMma 3.3. Suppose $X, Z$ are independent Poisson random variables with parameter $a \geq b$. Then

$$
\begin{aligned}
P\{X-Z=n\}=\frac{1}{\sqrt{a+b}}\left[\eta\left(\frac{n-(a-b)}{\sqrt{a+b}}\right)\right. & +O\left(b^{-1 / 2}\right)\left|\frac{n-(a-b)}{\sqrt{a+b}}\right|^{4} \\
& \left.\times \eta\left(\frac{n-(a-b)}{\sqrt{a+b}}\right)+o\left(b^{-2}\right)\right] .
\end{aligned}
$$

The following are easy corollaries of Chernoff's theorem [see Section 9 of Billingsley (1995)].

LEMMA 3.4. For every $\varepsilon>0$, there exist positive constants $c_{1}$ and $c_{2}$ such that if $X$ is a Poisson random variable with parameter $m$,

$$
P\{|X-m| \geq \varepsilon m\} \leq c_{1} \exp \left(-c_{2} m\right)
$$

The following result is straightforward from Lemma 3.4. 
COROLlaRY 3.5. There exist positive constants $c_{1}$ and $c_{2}$, such that if $Y$ is Poisson with parameter $a, Z$ is Poisson with parameter $b$ and $Y$ and $Z$ are independent,

$$
P\{Y-Z=0\} \leq c_{1} \exp \left(-c_{2}|a-b|\right)
$$

and

$$
P\{Y-Z=1\} \leq c_{1} \exp \left(-c_{2}|a-b|\right)
$$

4. Proofs of Theorems 2.1 and 2.3. In this section we will prove Theorems 2.1 and 2.3 as stated in Section 2. Before commencing with these proofs we must define several additional random variables and then state and prove two necessary lemmas.

First, we define two Poisson processes, $N_{q}(x)$ and $N_{p}(x)$, as follows:

$$
N_{q}(x)=\sup \left\{n>0: Q_{1}+\cdots+Q_{n} \leq x\right\}
$$

and

$$
N_{p}(x)=\sup \left\{n>0: P_{1}+\cdots+P_{n} \leq x\right\}
$$

where $Q_{i}$ and $P_{i}$ are as defined by (5) and (6), respectively. Note that for fixed $x, N_{q}(x)$ and $N_{p}(x)$ are Poisson random variables with rates $k_{2} x$ and $k_{1} x$, respectively. Now if we let $Y_{x}^{-1}$ be the amount of time needed for a particle to spend time $x$ in state $q$, then $Y_{x}^{-1}$ is the inverse process of $Y_{t}$ as defined in Section 2. Furthermore, $Y_{x}^{-1}=x+P_{1}+\cdots+P_{N_{q}(x)}$.

\section{LEMMA 4.1.}

$$
\begin{aligned}
f_{Y(t)}(x) & =\frac{d}{d x} P\left\{Y_{t} \leq x\right\} \\
& =k_{1} P\left\{N_{q}(x)-N_{p}(t-x)=1\right\}+k_{2} P\left\{N_{q}(x)-N_{p}(t-x)=0\right\} .
\end{aligned}
$$

Proof. By definition,

$$
f_{Y(t)}(x)=\lim _{\Delta x \rightarrow 0} \frac{P\left\{Y_{x}^{-1} \leq t\right\}-P\left\{Y_{x+\Delta x}^{-1} \leq t\right\}}{\Delta x} .
$$

Notice that

$$
\begin{aligned}
P\left\{Y_{x}^{-1}\right. & \leq t\}-P\left\{Y_{x+\Delta x}^{-1} \leq t\right\} \\
= & \sum_{n=0}^{\infty} P\left\{P_{1}+\cdots+P_{n} \leq t-x\right\} P\left\{N_{q}(x)=n\right\} \\
& -\sum_{n=0}^{\infty} P\left\{P_{1}+\cdots+P_{n} \leq t-(x+\Delta x)\right\} P\left\{N_{q}(x+\Delta x)=n\right\} .
\end{aligned}
$$


After adding and subtracting $\sum_{n=0}^{\infty} P\left\{P_{1}+\cdots+P_{n} \leq t-(x+\Delta x)\right\} P\left\{N_{q}(x)=n\right\}$ from the above equation, it is then straightforward to show that

$$
\begin{aligned}
& P\left\{Y_{x}^{-1} \leq t\right\}-P\left\{Y_{x+\Delta x}^{-1} \leq t\right\} \\
& =\sum_{n=0}^{\infty} P\left\{N_{q}(x)=n\right\}\left(P\left\{P_{1}+\cdots+P_{n} \leq t-x\right\}\right. \\
& \left.\quad-P\left\{P_{1}+\cdots+P_{n} \leq t-(x+\Delta x)\right\}\right) \\
& +\sum_{n=0}^{\infty}\left(P\left\{P_{1}+\cdots+P_{n} \leq t-(x+\Delta x) \leq P_{1}+\cdots+P_{n+1}\right\}\right. \\
& \left.\quad \times P\left\{N_{q}(x)=n, N_{q}(x+\Delta x)=n+1\right\}\right) \\
& +O\left((\Delta x)^{2}\right) .
\end{aligned}
$$

Since $N_{q}$ is a Poisson process with parameter $k_{2}$, it is easy to show that (17)

$$
P\left\{N_{q}(x)=n, N_{q}(x+\Delta x)=n+1\right\}=k_{2} \Delta x \exp \left(-k_{2} \Delta x\right) P\left\{N_{q}(x)=n\right\} .
$$

Recognizing that

$$
\begin{aligned}
& P\left\{P_{1}+\cdots+P_{n} \leq t-(x+\Delta x) \leq P_{1}+\cdots+P_{n+1}\right\} \\
& \quad=P\left\{N_{p}(t-(x+\Delta x))=n\right\}
\end{aligned}
$$

and using (17) and (16) in (15), we find

$$
f_{Y(t)}(x)=\sum_{n=0}^{\infty}\left(f_{P(1)+\cdots+P(n)}(t-x)+k_{2} P\left\{N_{p}(t-x)=n\right\}\right) P\left\{N_{q}(x)=n\right\} .
$$

Finally, noticing that

$$
f_{P(1)+\cdots+P(n)}(t-x)=\frac{k_{1} \exp \left(-k_{1}(t-x)\right)\left(k_{1}(t-x)\right)^{n}}{n !}=k_{1} P\left\{N_{p}(t-x)=n-1\right\}
$$

completes the proof of Lemma 4.1.

LEMMA 4.2. For any $x>0$,

$$
\begin{gathered}
\frac{k_{1}+k_{2}}{\sqrt{k_{1}(t-x)+k_{2} x}} \eta\left(\frac{k_{1}(t-x)-k_{2} x}{\sqrt{k_{1}(t-x)+k_{2} x}}\right) \\
=\left(1+O\left(t^{-1 / 2}\right)\right) \frac{1}{\sigma \sqrt{t}} \eta\left(\frac{x-\mu t}{\sigma \sqrt{t}}\right),
\end{gathered}
$$

where $\mu=k_{1}\left(k_{1}+k_{2}\right)^{-1}$ and $\sigma^{2}=2 k_{1} k_{2}\left(k_{1}+k_{2}\right)^{-3}$.

Proof. Assume $x=\mu t+b \sqrt{t}$, where $b$ is a constant.

Case $1(b=0)$. First we suppose $b=0$, and thus $x=\mu t$. Then

$$
\begin{aligned}
\frac{k_{1}+k_{2}}{\sqrt{k_{1}(t-x)+k_{2} x}} \eta\left(\frac{k_{1}(t-x)-k_{2} x}{\sqrt{k_{1}(t-x)+k_{2} x}}\right) & =\frac{k_{1}+k_{2}}{\sqrt{2 \pi 2 k_{1} k_{2}\left(k_{1}+k_{2}\right)^{-1} t}} \\
& =\frac{1}{\sigma \sqrt{t}} \eta\left(\frac{x-\mu t}{\sigma \sqrt{t}}\right) .
\end{aligned}
$$


Case $2(b \neq 0)$. Now suppose that $x=\mu t+b \sqrt{t}$, where $b \neq 0$. We can easily show that

$$
\begin{aligned}
\eta\left(\frac{k_{1}(t-x)-k_{2} x}{\sqrt{k_{1}(t-x)+k_{2} x}}\right) & =\eta\left(\frac{x-\mu t}{\sigma \sqrt{t}}\right) \exp \left(O\left(t^{-1 / 2}\right)\right) \\
& =\eta\left(\frac{x-\mu t}{\sigma \sqrt{t}}\right)\left(1+O\left(t^{-1 / 2}\right)\right)
\end{aligned}
$$

It is also easy to show that

$$
\begin{aligned}
\frac{k_{1}+k_{2}}{\sqrt{k_{1}(t-x)+k_{2} x}} & =\frac{k_{1}+k_{2}}{\sqrt{2 k_{1} k_{2} t\left(k_{1}+k_{2}\right)^{-1}}}+O\left(t^{-1}\right) \\
& =\frac{1}{\sigma \sqrt{t}}+O\left(t^{-1}\right) .
\end{aligned}
$$

Using (19) and (20) we find that

$$
\begin{aligned}
& \frac{k_{1}+k_{2}}{\sqrt{k_{1}(t-x)+k_{2} x}} \eta\left(\frac{k_{1}(t-x)-k_{2} x}{\sqrt{k_{1}(t-x)+k_{2} x}}\right) \\
& =\left(\frac{1}{\sigma \sqrt{t}}+O\left(t^{-1}\right)\right) \eta\left(\frac{x-\mu t}{\sigma \sqrt{t}}\right)\left(1+O\left(t^{-1 / 2}\right)\right) \\
& =\left(1+O\left(t^{-1 / 2}\right)\right) \frac{1}{\sigma \sqrt{t}} \eta\left(\frac{x-\mu t}{\sigma \sqrt{t}}\right) .
\end{aligned}
$$

This completes the proof of Lemma 4.2 .

We now have the tools to prove our main results.

Proof of Theorem 2.1. Assume $x \in[\mu t / 2,(\mu+1) t / 2]$. We need to show that

$$
\begin{aligned}
f_{Y(t)}(x)=(1 & \left.+O\left(t^{-1 / 2}\right)\right) \frac{1}{\sigma \sqrt{t}} \eta\left(\frac{x-\mu t}{\sigma \sqrt{t}}\right)+\frac{1}{\sqrt{\left.k_{1}(t-x)+k_{2} x\right)}} O\left(t^{-1 / 2}\right) \\
& \times\left|\frac{k_{1}(t-x)-k_{2} x}{\sqrt{k_{2} x+k_{1}(t-x)}}\right|^{4} \eta\left(\frac{k_{1}(t-x)-k_{2} x}{\sqrt{k_{2} x+k_{1}(t-x)}}\right) \\
& +o\left(t^{-3 / 2}\right) .
\end{aligned}
$$

By Lemma 4.1 we know that

$$
f_{Y(t)}(x)=k_{1} P\left\{N_{q}(x)-N_{p}(t-x)=1\right\}+k_{2} P\left\{N_{q}(x)-N_{p}(t-x)=0\right\} .
$$

Recall that $N_{q}(x)$ and $N_{p}(t-x)$ are Poisson r.v.'s with parameters $k_{2} x$ and $k_{1}(t-x)$, respectively. Hence, we may use Lemma 3.3 , the local central 
limit theorem estimate for differences of Poisson r.v.'s, to conclude that for $x \in[\mu t / 2,(\mu+1) t / 2]$,

$$
\begin{aligned}
k_{1} P\left\{N_{q}(x)-N_{p}(t-x)=\right. & 1\} \\
=\frac{k_{1}}{\sqrt{k_{1}(t-x)+k_{2} x}}[ & \eta\left(\frac{1-\left(k_{2} x-k_{1}(t-x)\right)}{\sqrt{k_{1}(t-x)+k_{2} x}}\right) \\
& +O\left(t^{-1 / 2}\right)\left|\frac{1-\left(k_{2} x-k_{1}(t-x)\right)}{\sqrt{k_{1}(t-x)+k_{2} x}}\right|^{4} \\
& \left.\times \eta\left(\frac{1-\left(k_{2} x-k_{1}(t-x)\right)}{\sqrt{k_{1}(t-x)+k_{2} x}}\right)+o\left(t^{-2}\right)\right] \\
=\frac{k_{1}}{\sqrt{k_{1}(t-x)+k_{2} x}}[ & \eta\left(\frac{k_{1}(t-x)-k_{2} x}{\sqrt{k_{1}(t-x)+k_{2} x}}\right) \\
& +O\left(t^{-1 / 2}\right)\left|\frac{k_{1}(t-x)-k_{2} x}{\sqrt{k_{1}(t-x)+k_{2} x}}\right|^{4} \\
& \left.\times \eta\left(\frac{k_{1}(t-x)-k_{2} x}{\sqrt{k_{1}(t-x)+k_{2} x}}\right)+o\left(t^{-2}\right)\right]
\end{aligned}
$$

Similarly, for $x \in[\mu t / 2,(\mu+1) t / 2]$,

$$
\begin{aligned}
k_{2} P\left\{N_{q}(x)-N_{p}(t-x)=\right. & 0\} \\
=\frac{k_{2}}{\sqrt{k_{1}(t-x)+k_{2} x}}[ & \eta\left(\frac{k_{1}(t-x)-k_{2} x}{\sqrt{k_{1}(t-x)+k_{2} x}}\right) \\
& +O\left(t^{-1 / 2}\right)\left|\frac{k_{1}(t-x)-k_{2} x}{\sqrt{k_{1}(t-x)+k_{2} x}}\right|^{4} \\
& \left.\times \eta\left(\frac{k_{1}(t-x)-k_{2} x}{\sqrt{k_{1}(t-x)+k_{2} x}}\right)+o\left(t^{-2}\right)\right] .
\end{aligned}
$$

Adding (22) and (23) and using Lemma 4.2, we find that

$$
\begin{aligned}
f_{Y(t)}(x)= & \left(1+O\left(t^{-1 / 2}\right)\right) \frac{1}{\sigma \sqrt{t}} \eta\left(\frac{x-\mu t}{\sigma \sqrt{t}}\right) \\
& +\frac{1}{\sqrt{\left.k_{1}(t-x)+k_{2} x\right)}} O\left(t^{-1 / 2}\right)\left|\frac{k_{1}(t-x)-k_{2} x}{\sqrt{k_{2} x+k_{1}(t-x)}}\right|^{4} \\
& \times \eta\left(\frac{k_{1}(t-x)-k_{2} x}{\sqrt{k_{2} x+k_{1}(t-x)}}\right) \\
& +o\left(t^{-3 / 2}\right) .
\end{aligned}
$$

This completes the proof of Theorem 2.1. 
Since $X_{t}=v Y_{t}$, Corollary 2.2 follows immediately.

Proof of Theorem 2.3. Let $x=\mu v T+a \sqrt{T}$. Recall from Section 2 that

$$
p(x, T)+q(x, T)=q_{0} v \int_{0}^{T} f_{X(t)}(x) d t .
$$

For $x \in[\mu v t / 2,(\mu+1) v t / 2], f_{X(t)}(x)$ is given by Corollary 2.2. Since $X_{t}=v Y_{t}$ and

$$
f_{Y(t)}(x)=k_{1} P\left\{N_{q}(x)-N_{p}(t-x)=1\right\}+k_{2} P\left\{N_{q}(x)-N_{p}(t-x)=0\right\},
$$

we can apply the large deviation estimate given in Corollary 3.5 to conclude that

$$
f_{Y(t)}(x) \leq \begin{cases}c_{1} \exp \left(-c_{3} t\right), & \text { if } x<\mu t / 2, \\ c_{1} \exp \left(-c_{4} t\right), & \text { if } x>(\mu+1) t / 2\end{cases}
$$

for some positive constants $c_{1}, c_{3}$, and $c_{4}$.

Letting $b=2 x((\mu+1) v)^{-1}, c=\min \left(2 x(\mu v)^{-1}, T\right)$ and applying (25) and Corollary 2.2 to (24), we find that

$$
\begin{aligned}
p(x, T)+q(x, T)= & q_{0}\left(1+O\left(T^{-1 / 2}\right)\right) \int_{b}^{c} \frac{1}{\sigma \sqrt{t}} \eta\left(\frac{x v^{-1}-\mu t}{\sigma \sqrt{t}}\right) d t \\
+ & O\left(T^{-1}\right) \int_{b}^{c}\left|\frac{k_{1}\left(t-x v^{-1}\right)-k_{2} x v^{-1}}{\sqrt{k_{2} x v^{-1}+k_{1}\left(t-x v^{-1}\right)}}\right|^{4} \\
& \times \eta\left(\frac{k_{1}\left(t-x v^{-1}\right)-k_{2} x v^{-1}}{\sqrt{k_{2} x v^{-1}+k_{1}\left(t-x v^{-1}\right)}}\right) d t+o\left(T^{-1 / 2}\right),
\end{aligned}
$$

Using standard analysis techniques it can be shown that

$$
\begin{aligned}
& O\left(T^{-1}\right) \int_{b}^{c}\left|\frac{k_{1}\left(t-x v^{-1}\right)-k_{2} x v^{-1}}{\sqrt{k_{2} x v^{-1}+k_{1}\left(t-x v^{-1}\right)}}\right|^{4} \eta\left(\frac{k_{1}\left(t-x v^{-1}\right)-k_{2} x v^{-1}}{\sqrt{k_{2} x v^{-1}+k_{1}\left(t-x v^{-1}\right)}}\right) d t \\
& \quad=O\left(T^{-1 / 2}\right)
\end{aligned}
$$

and that

$$
\begin{aligned}
q_{0} \int_{b}^{c} & \frac{1}{\sigma \sqrt{t}} \eta\left(\frac{x v^{-1}-\mu t}{\sigma \sqrt{t}}\right) d t \\
= & q_{0} \mu^{-1} \int_{-\infty}^{0} \frac{1}{\sqrt{2 \pi \sigma^{2} T}} \exp \left(\frac{-\left(x v^{-1}-\mu T-y\right)^{2}}{2 \sigma^{2} T}\right) \\
& +O\left(T^{-1}\right) .
\end{aligned}
$$

Substituting (27) and (28) into (26), we find that

$$
\begin{aligned}
p(x, T)+q(x, T)= & q_{0} \mu^{-1} \int_{-\infty}^{0} \frac{1}{\sqrt{2 \pi \sigma^{2} T}} \exp \left(\frac{-\left(x v^{-1}-\mu T-y\right)^{2}}{2 \sigma^{2} T}\right) d y \\
& +O\left(T^{-1 / 2}\right) \\
= & q_{0} \mu^{-1} H\left(v^{-1}(x-\mu v T), T\right)+O\left(T^{-1 / 2}\right),
\end{aligned}
$$


where

$$
H(s, t)=\int_{-\infty}^{0} \frac{1}{\sqrt{2 \pi \sigma^{2} t}} \exp \left(\frac{-(s-y)^{2}}{2 \sigma^{2} t}\right) d y
$$

is the solution of the following initial value problem for the heat equation

$$
\begin{aligned}
\frac{\partial H}{\partial t} & =\frac{1}{2} \sigma^{2} \frac{\partial^{2} H}{\partial s^{2}} \\
H(s, 0) & = \begin{cases}1, & \text { for } s \leq 0 \\
0, & \text { for } s>0\end{cases}
\end{aligned}
$$

This completes the proof of Theorem 2.3.

Acknowledgment. The work presented in this paper is a portion of a Ph.D. thesis written under the direction of Michael C. Reed and Gregory F. Lawler at Duke University.

\section{REFERENCES}

BARTLett, M. (1957). Some problems associated with random velocity. Publ. Inst. Statist. Univ. Paris 6 261-270.

Bartlett, M. (1978). A note on random walks at constant speed. Adv. Appl. Probab. 10 704-707. Billingsley, P. (1995). Probability and Measure. Wiley, New York.

Blum, J. J. and ReED, M. C. (1985). A model for fast axonal transport. Cell Motility 5 507-527.

CANE, V. (1967). Random walks and physical processes. Bull. Internat. Statist. Inst. 42 622-640.

CANE, V. (1975). Diffusion models with relativity effects. In Perspectives of Probability and Statistics (J. Gani, ed.) 263-273. Applied Probability Trust, Sheffield.

Cox, D. R. (1967). Renewal Theory. Methuen, London.

FelleR, W. (1968). An Introduction to Probability Theory and Its Applications. Wiley, New York. GoldSTEIN, S. (1951). On diffusion by discontinuous movements and the telegraph equation. Quart. J. Mech. Appl. Math. 4 129-156.

Ibragimov, I. A. and LINNIK, Y. V. (1971). Independent and Stationary Sequences of Random Variables. Wolters-Noordhoff, Groningen.

KAC, M. (1974). A stochastic model related to the telegrapher's equation. Rocky Mountain J. Math. 4 497-509.

KAPLAN, S. (1964). Differential equations in which the poisson process plays a role. Bull. Amer. Math. Soc. 70 264-268.

Karlin, S. and TaYlor, H. M. (1975). A First Course in Stochastic Processes. Academic Press, San Diego.

KolesniK, A. (1998). The equations of Markovian random evolution in a line. J. Appl. Probab. 35 27-35.

LAWleR, G. F. (1995). Introduction to Stochastic Processes. Chapman and Hall, New York.

ORsINGHER, E. (1985). Hyperbolic equations arising in random models. Stochastic Process. Appl. 21 93-106.

Orsingher, E. (1990). Random motions governed by third-order equations. Adv. Appl. Probab. 22 915-928.

ORSINGHER, E. and BASSAN, B. (1992). On a $2 n$-valued telegraph signal and the related integrated process. Stochastics Rep. 38 159-173. 
REED, M. C. and BLum, J. J. (1986). Theoretical analysis of radioactivity profiles during fast axonal transport: effects of deposition and turnover. Cell Motility and the Cytoskeleton 6 620-627.

Reed, M. C., Venakides, S. and Blum, J. J. (1990). Approximate traveling waves in linear reaction-hyperbolic equations. SIAM J. Appl. Math. 50 167-180.

LABORATORY OF COMPUTATIONAL BIOLOGY AND RISK ANALYSIS National Institute of Environmental Health Sciences

P.O. Box 12233

Research Triangle Park, North Carolina 27709-2233

E-MAIL: EABrooks2@aol.com 